\title{
Silencing NACK by siRNA inhibits tumorigenesis in non-small cell lung cancer via targeting Notch1 signaling pathway
}

\author{
RANRAN KONG $^{1}$, JIE FENG $^{2}$, YUEFENG MA ${ }^{1}$, BIN ZHOU $^{1}$, SHAOMIN LI $^{1}$, WEI ZHANG ${ }^{1}$, JIANTAO JIANG $^{1}$, \\ JIN ZHANG ${ }^{1}$, ZHE QIAO $^{1}$, TING ZHANG ${ }^{3}$, QUANJIN ZANG ${ }^{3}$ and XIJING HE ${ }^{3}$ \\ ${ }^{1}$ Department of Thoracic Surgery, The Second Affiliated Hospital of Xi'an Jiaotong University, Xi'an, Shaanxi 710004; \\ ${ }^{2}$ Department of Nephrology, The First Affiliated Hospital of Xi'an Jiaotong University, Xi'an, Shaanxi 710061; \\ ${ }^{3}$ Second Department of Orthopedics, The Second Affiliated Hospital \\ of Xi'an Jiaotong University, Xi'an, Shaanxi 710004, P.R. China
}

Received October 30, 2015; Accepted December 8, 2015

DOI: $10.3892 /$ or.2016.4552

\begin{abstract}
Non-small cell lung cancer (NSCLC) is the most common type of lung tumor with poor prognosis, in which the Notch signaling pathway plays an important role. Notch activation complex kinase (NACK) has been reported both as a co-activator and a target gene of the Notch pathway. However, the molecular mechanism of NACK in NSCLC still remains unknown. In this study, the expression of NACK was analyzed in 35 paired NSCLC tumor samples and 2 NSCLC cell lines. MTT assay, cell migration assay, cell invasion assay, flow cytometry assay, and xenograft model were employed to detect the effect of NACK knockdown on the cell proliferation, metastasis, invasion and apoptosis of NSCLC. The relationship between NACK and Notch1 signaling pathway in NSCLC cells was assessed by western blot and luciferase reporter assay. We found that the expression of NACK in the NSCLC tissues and lung cancer cells were significantly increased. High level of NACK expression is remarkable associated with tumor differentiation, lymphatic metastasis, clinical stage and poor survival prognosis. The interference of NACK significantly inhibited cell proliferation, invasion and metastasis through inducing apoptosis in NSCLC cells. The transcriptional activity of related Notch1 target genes were significantly suppressed resulting from NACK knockdown. This study demonstrates that interference of NACK inhibits NSCLC progression through Notch1 signaling pathway and targeting NACK may be an effective approach for NSCLC therapy.
\end{abstract}

Correspondence to: Dr Xijing He, Second Department of Orthopedics, The Second Affiliated Hospital of Xi'an Jiaotong University, Xi'an, Shaanxi 710004, P.R. China

E-mail: zhoubineryuan@163.com

Key words: Notch activation complex kinase, non-small cell lung cancer, cell proliferation, cell apoptosis, Notch1, poor prognosis

\section{Introduction}

Lung cancer is one of the leading causes of cancer-related mobility worldwide affecting millions of people every year $(1,2)$. Lung cancer consists of two major types: small cell lung cancer (SCLC) and non-small cell lung cancer (NSCLC), of which NSCLC account for $80 \%$ of them (3). Though various therapeutic agents for the treatment of NSCLC have been developed (4), poor prognosis was always discovered in patients on account of the frequent metastasizing characteristic of this malignancy (5).

The Notch signaling pathway has been identified as critical in governing cell fate determination by directly regulating transcriptional programs, including differentiation, proliferation, self-renewal, and apoptosis (6-8). Notch-1 signaling is activated when the complex of ligand and receptor binding is formed between directly contacting cells $(9,10)$. Then Notch1 is cleaved by r-secretase and translocates the Notch-1 intracellular domain (NICD) from the plasma membrane into the nucleus to regulate related target genes including hairy enhance of split (Hes) family and hairy enhancer of split related with YRPW motif (Hey) family $(11,12)$. Previous studies have reported that aberrant activation of Notch could cause a variety of solid malignancies including ovarian, breast, lung, and renal cancer, suggesting Notch may be an attractive target for therapeutic intervention in cancer $(13,14)$.

Notch activation complex kinase (NACK) is the first identified as both a co-activator and a target gene of the Notch pathway. NACK regulates Notch transcriptional activity by interaction with the Notch transcriptional activation complex on DNA, which is required for Notch-mediated tumorigenesis (15). Recent studies have showed that knockdown of NACK resulted in inhibiting tumor growth in human esophageal carcinoma cells (15). However, the role and underlying mechanism of NACK in NSCLC still remains unknown.

In the present study, we analyzed the expression of NACK among NSCLC patients, and further investigated the role of NACK on tumor growth of NSCLC by small RNA interference method both in vitro and in vivo. We found that NACK expression was significantly increased in NSCLC tumors and cell lines. High NACK expression was associated with 
different clinicopathological parameters and poor prognosis. Knocking down NACK could directly inhibit cell proliferation and increase cell apoptosis. Downregulation of Hes1, HeyL and Notch1 was discovered in the NACK knockdown in NSCLC cells. Our study may provide a better understanding of the underlying molecular mechanism of NACK in the regulation of NSCLC.

\section{Materials and methods}

Tissue sample collection. In this study, NSCLC tumor tissues and paired adjacent normal tissues were obtained from 35 patients who underwent percutaneous lung puncture or biopsy of lung cancer tissue at The Second Affiliated Hospital of Xi'an Jiaotong University (Xi'an, China) between October 2013 and May 2014. All NSCLC cases were clinically and pathologically confirmed by two independent experts. All tissues were frozen at $-80^{\circ} \mathrm{C}$ for further analysis. The follow-up data for 60 months were recorded by communicating with the patients or their relatives, the median duration of follow-up was 59 months (range, 1-60 months). Informed consent was obtained from each patient, and this study was approved by the Human Ethics Committee of Second Affiliated Hospital of Xi'an Jiaotong University.

Cell lines and cell culture. The human lung adenocarcinoma cell lines A549 and H1299 and normal human bronchial epithelial cell line (NHBE) were obtained from the Shanghai GeneChem Co., Bank (Shanghai, China). All cell culture reagents were purchased from Invitrogen and supplied with $1 \%$ penicillin/streptomycin (Sigma-Aldrich, ON, Canada). Cells were grown in Dulbecco's modified Eagle's medium (DMEM) supplemented with 10\% FBS (Hyclone, Logan, UT, USA), and maintained in monolayer culture at $37^{\circ} \mathrm{C}$ in an incubator of humidified air with $5 \% \mathrm{CO}_{2}$.

Plasmid construction and transient transfection of NSCLC cell lines. For NACK, the sequences for siRNA were design as follows: sense strand, 5'-UCG CAU UGA CCA UUC AAA CUG GUG G-3' and antisense, 5'-CCA CCA GUU UGA AUG GUC AAU GCG A-3'. The scramble siRNA was random sequenced by Blast website. The cells were harvested at $48 \mathrm{~h}$ post-transfection. A549 and H1299 cells were used in this experiment. Twenty-four hours before transfection, $1 \times 10^{4}$ cells/well, in $5 \mathrm{ml}$ medium, were plated in $60 \mathrm{~mm}$ dish (Nunc; Thermo Fisher Scientific, Waltham, MA, USA), and then transfected with plasmids of NACK-siRNA or scramble siRNA as negative control using Lipofectamine 2000 as described in the manufacturer's instruction.

Luciferase reporter assay. For reporter assay, A549 and H1299 cells were cultured in 24-well plates. Hes1-luciferase reporter plasmids were constructed by subcloning the 5-upsteam elements of human Hes1 into the pGL3-enhancer vector (Promega, Madison, WI, USA). Hes1 promoter-specific primers (-747-+66): Forward, 5'-CGA GCT CAG CGG CAA CTT TAG ATG TG-3'; and reverse, 5'-CCC AAG CTT GTT GAC ACT GGC TGG GGT A-3'. After $24 \mathrm{~h}$, the cells were co-transfected with the siRNA expression plasmid targeting NACK (NACK siRNA). Fourty-eight hours later, luciferase activities were measured using Dual-Luciferase Reporter Assay system (Promega) according to manufacturer's instructions. Firefly luciferase activity was normalized to Renilla luciferase activity.

RNA extraction and quantitative RT-PCR analysis. Total RNA was isolated from cells or tissues by TRIzol reagent (15596-026; Life Technologies) following the manufacturer's instructions. Quantitative real-time reverse transcriptase-PCR assay (qRT-PCR) was performed using a SYBR-Green method with a Master Mix buffer system. Gene expression in human was normalized to GAPDH. The primers sequences were as follows: GAPDH forward, 5'-CCG ATT TCT CCT CCG GGT G-3' and reverse, 5'-TGG TCA TGA GTC CTT CCA CG-3'; NACK forward, 5'-TCT CTT GTG AAG GAA CCG GC-3' and reverse, 5'-CCG GCT TGT AAG TCC TGG TT-3'; Notch1 forward, 5'-GGG CCT CAA GTG AGC GGA C-3' and reverse, 5'-GGT GAG GGG TCG AGA AGT GA-3'; Hes1 forward, 5'-TTT CTT CCA GAC TTC CGC CC-3' and reverse, 5'-GGA CAA TGC CTC CCA ATC CA-3'; and HeyL forward, 5'-AGA CCG CAT CAA CAG TAG CC-3' and reverse, 5'-TCA GGC AGC TGC TAC CAA TC-3'. The PCR conditions were as follows: $95^{\circ} \mathrm{C}$ for $2 \mathrm{~min}, 95^{\circ} \mathrm{C}$ for $15 \mathrm{sec}, 60^{\circ} \mathrm{C}$ for $30 \mathrm{sec}$ for 40 cycles. The relative expression levels were calculated by the value of $2^{-\Delta \Delta C t}$. All experiments were repeated at least three times.

Western blotting. The tissues and cells were washed twice with ice-cold PBS and lysed in RIPA lysis buffer (Solarbio, Beijing, China). The proteins were separated on SDS-polyacrylamide gels (SDS-PAGE) and electrophoretically transferred to PVDF membranes (Roche Diagnostics). Then the membranes were blocked in $5 \%$ milk, and incubated with the appropriate primary antibody overnight. The primary antibodies NACK (1:1,000; AbMax Company, China), Notch1 (1:1,000), Hes1 $(1: 1,000)$, and HeyL $(1: 1,000)$ (all from Abcam, UK), were incubated with HRP-conjugated secondary antibodies (Abcam) at 1:1,000 for $1 \mathrm{~h}$, then the protein bands were detected using the enhanced chemiluminescence detection system (Pierce; Thermo Scientific, Rockford, IL, USA).

Cell proliferation assay. The proliferation of NSCLC cells was evaluated by Cell Counting Kit-8 (CCK-8) assay (Beyotime, Shanghai, China) according to manufacturer's instruction. Briefly, cells transfected with negative-siRNA or NACK-siRNA were seeded into a 96-well plate $(100 \mu \mathrm{l} /$ well $)$ and incubated at $37^{\circ} \mathrm{C}, 5 \% \mathrm{CO}_{2}$ for 5 days. CCK-8 (10\%) was added to the well at $24 \mathrm{~h}$ intervals and maintained for $2 \mathrm{~h}$ according to the manufacturer's protocol, and then the absorbance was measured at $450 \mathrm{~nm}$ by a microplate reader (BioTek ELx800; BioTek, USA).

Colony formation assay. Non-small cell lung cancer cells (A549 and H1299) were transfected with negative-siRNA or NACK-siRNA for $24 \mathrm{~h}$, then the transfected cells were resuspended, counted and seeded at a density of 600 cells/well in a 6-well plate. The cells were continually cultured for 10 days with a change of media every other day. On day 10 , cells were fixed with $3.7 \%$ methanol, stained with $0.1 \%$ crystal violet the number of colonies were counted under a microscope (Olympus, Beijing, China). 

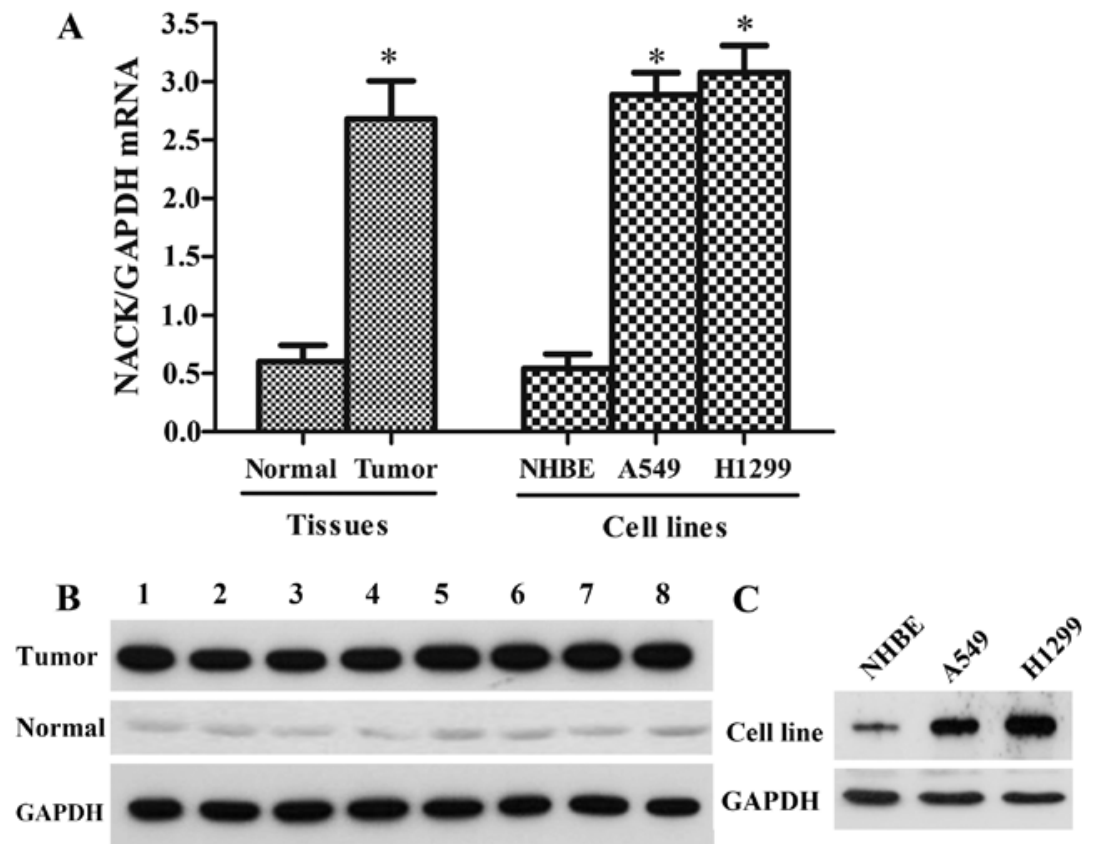

Figure 1. Expression of NACK in NSCLC tumors. (A) The relative mRNA level of NACK among 35 paired tumor tissues and 3 lung cell lines. (B) Western blot analyses the expression of NACK in 8 representative tumor tissues and their paired adjacent normal tissues. (C) Western blot analyses the expression of NACK in 3 lung cell lines. GAPDH was used as internal control. Data shown as mean \pm SD of triplicate independent experiments. " $p<0.05$ when compared to negative-siRNA group.

are of Migration and invasion assays. Cell motility was assessed by migration and invasion assays. A 24-well Transwell insert containing a pore size of $8 \mu \mathrm{m}$ polycarbonate membrane (Corning, Inc., Corning, NY, USA) was used to determine the effect of NACK on A549 and H1299 migration and invasion in vitro. Briefly, the transfected cells were cultured in serum-free medium for $12 \mathrm{~h}$, then cells were resuspended in serum-free medium and placed in the upper chambers at the density of $2 \times 10^{4}$. The lower chamber was filled with medium containing $10 \%$ fetal bovine serum as the chemoattractant and incubated $24 \mathrm{~h}$ for migration assay and $48 \mathrm{~h}$ for the invasion assay, respectively. For migration assay, the number of cells on the lower surface of the membrane was counted in 5 fields under a microscope. For invasion assay, the upper chambers of the inserts were covered with Matrigel. After $48 \mathrm{~h}$ incubation, cells on the lower surface of the member was stained with $0.1 \%$ crystal violet. The number of cells on the lower surface of the membrane was counted in 5 fields under a microscope.

Apoptosis. NSCLC cells transfected as negative-siRNA or NACK-siRNA were collected and washed with PBS twice. After centrifuge, the cells were resuspended with $1 \mathrm{X}$ staining buffer at the dose of $1 \times 10^{6} \mathrm{cell} / \mathrm{ml}$, then cells were dyed with $5 \mu 1$ Annexin V-APC in the dark, at room temperature for 15 min. Flow cytometry was applied with FACSCalibur flow cytometer and analysis was performed with FlowJo software (Tree Star).

Xenografts. Four weeks old female nude mice were purchased from the Animal Center of The Fourth Military Medical University (Xi'an, China) and randomly divided into three groups with 10 per group, receiving subcutaneous injection of NACK-siRNA, negative-siRNA, or control cells, respectively. of Tumor volume was measured using calipers every 4 days for 28 days and calculated as follows: Tumor size [volume $\left(\mathrm{mm}^{3}\right)=$ width $^{2}(\mathrm{~mm}) / 2 \mathrm{x}$ length $\left.(\mathrm{mm})\right]$. Following 28-day post-inoculation, the animals were euthanized and the tumors were collected for further analyzing. The animal experiment was reviewed and approved by the Animal Care and Use Committee of the Second Affiliated Xi'an Jiaotong University (Xi'an, China).

Statistical analysis. All assays were repeated three times to insure reproducibility. The significance of results obtained from the control and treated groups were analyzed using the Student's t-test. The results are given as mean \pm SD. $\mathrm{p}<0.05$ was considered statistically significant.

\section{Results}

Overexpression of NACK in NSCLC tumor tissues and cell lines. The expression level of NACK in 35 NSCLC tissue samples and corresponding adjacent non-cancerous tissues were detected by the quantitative RT-PCR (qRT-PCR) and western blot assay. As shown in Fig. 1A and B, NACK was discovered highly expressed both at mRNA and protein levels in the NSCLC tissue samples compared with the paired adjacent normal tissues. Furthermore, the expression level of NACK was assessed in NSCLC cell lines (A549 and H1299) and NHBE, the result showed that NACK was greatly higher in A549 and H1299 cell lines than the normal cell line NHBE (Fig. 1A and C), indicating that NACK may play a positive role in NSCLC progression.

High NACK expression is associated with different clinicopathological parameters. To determine the potential role of 
A

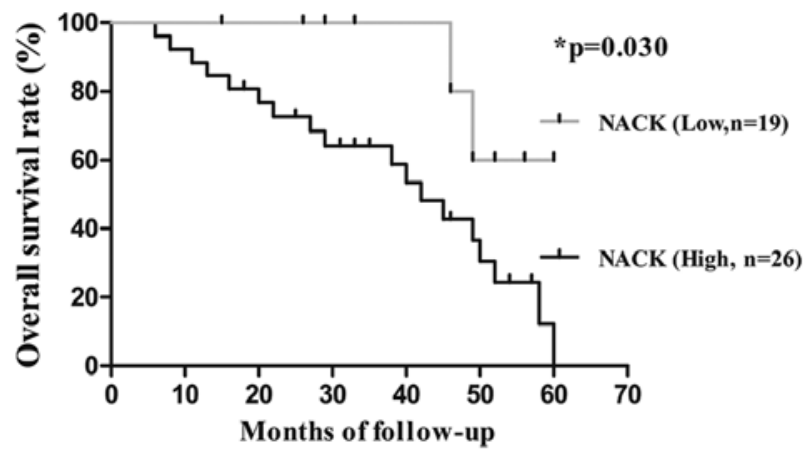

B

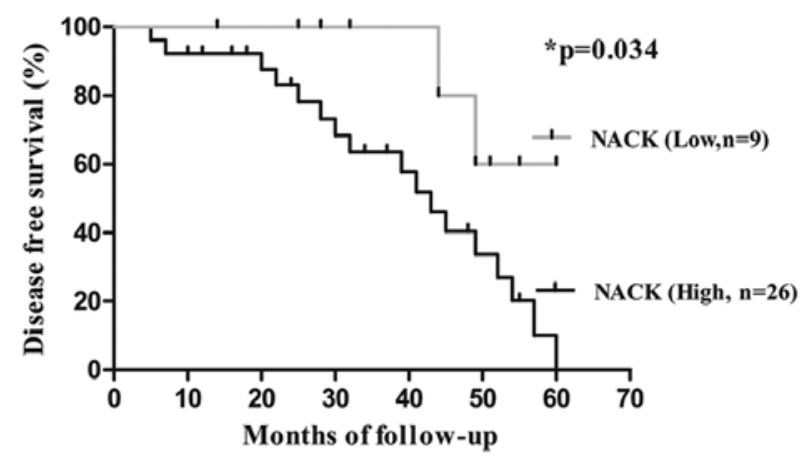

Figure 2. Kaplan-Meier survival curves for overall survival and disease-free survival stratified by NACK expression. (A) Overall survival for high and low NACK expression patients. (B) Disease-free survival for high and low NACK expression patients. Patients with high NACK expression have poorer overall survival and disease-free survival. " $\mathrm{p}<0.05$ statistically significant.

Table I. Association between NACK expression and clinicopathological factors in patient with NSCLC.

\begin{tabular}{|c|c|c|c|c|}
\hline \multirow[b]{2}{*}{ Variables } & \multirow{2}{*}{$\begin{array}{c}\text { No. } \\
\text { of cases }\end{array}$} & \multicolumn{2}{|c|}{ NACK expression } & \multirow[b]{2}{*}{ P-value } \\
\hline & & Low $(\%)$ & High (\%) & \\
\hline \multicolumn{5}{|l|}{ Gender } \\
\hline Male & 19 & $5(26.32)$ & $14(73.68)$ & \multirow[t]{2}{*}{0.471} \\
\hline Female & 16 & $4(25.00)$ & $12(75.00)$ & \\
\hline \multicolumn{5}{|l|}{ Age (years) } \\
\hline$>60$ & 24 & $5(20.83)$ & 19 (79.17) & \multirow[t]{2}{*}{0.583} \\
\hline$\leq 60$ & 11 & $4(36.36)$ & 7 (63.64) & \\
\hline \multicolumn{5}{|l|}{ Smoking } \\
\hline Yes & 22 & $4(18.18)$ & $18(81.82)$ & \multirow{2}{*}{0.192} \\
\hline No & 13 & $5(38.46)$ & $8(61.54)$ & \\
\hline \multicolumn{5}{|c|}{ Tumor size $(\mathrm{cm})$} \\
\hline$>3$ & 21 & $6(28.57)$ & $15(71.43)$ & \multirow[t]{2}{*}{0.538} \\
\hline$\leq 3$ & 14 & $3(21.43)$ & $11(78.57)$ & \\
\hline \multicolumn{5}{|c|}{ Histological type } \\
\hline $\mathrm{AC}$ & 26 & $6(23.08)$ & $20(76.92)$ & \multirow[t]{3}{*}{0.139} \\
\hline SCC & 7 & $2(28.57)$ & $5(71.43)$ & \\
\hline Other & 2 & $1(50.00)$ & $1(50.00)$ & \\
\hline \multicolumn{5}{|c|}{ Differentiation } \\
\hline Well & 11 & $4(36.36)$ & 7 (63.64) & \multirow[t]{3}{*}{$0.034^{\mathrm{a}}$} \\
\hline Moderate & 15 & $4(26.67)$ & $11(73.33)$ & \\
\hline Poor & 9 & $1(11.11)$ & $8(88.89)$ & \\
\hline \multicolumn{5}{|l|}{ T stage } \\
\hline $\mathrm{T} 1-2$ & 23 & $6(26.09)$ & $17(73.91)$ & \multirow[t]{2}{*}{0.316} \\
\hline T3-4 & 12 & $3(25.00)$ & $9(75.00)$ & \\
\hline \multicolumn{5}{|l|}{$\mathrm{N}$ stage } \\
\hline No & 17 & $5(29.41)$ & $12(70.59)$ & \multirow[t]{2}{*}{$0.007^{\mathrm{b}}$} \\
\hline $\mathrm{N} 1,2,3$ & 18 & $4(22.22)$ & 14 (77.78) & \\
\hline \multicolumn{5}{|c|}{ Clinical stage } \\
\hline I-II & 21 & $5(23.81)$ & $16(76.19)$ & \multirow[t]{2}{*}{$0.014^{\mathrm{a}}$} \\
\hline III-IV & 14 & $4(28.57)$ & $10(71.43)$ & \\
\hline
\end{tabular}

P-value ${ }^{\mathrm{a}}<0.05{ }^{\mathrm{b}}<0.01$ statistically significant.
NACK in the progress of NSCLC, the relationship between NACK expression and clinicopathological parameters in NSCLC were analyzed by Pearson's Chi-square. NSCLC cases were divided into two groups according to the mRNA level of NACK (low < median; high > median). The high expression of NACK was observed in 26/35 (74.29\%) of NSCLC samples, and the expression of NACK was significantly correlated with the degree of differentiation ( $\mathrm{p}=0.034)$, lymphatic metastasis (N stage) $(\mathrm{p}=0.007)$ and clinical stage $(\mathrm{p}=0.014)$ (Table $\mathrm{I})$. However, the high level of NACK expression was not associated with gender, age, smoking, tumor size or histological type $(p>0.05)$. Hence, NACK may be used as a potential unfavorable prognostic biomarker in NSCLC.

NACK as a prognostic marker in NSCLC patients. To investigate the correlation between NACK expression and the survival rates of NSCLC patients, Kaplan-Meier survival analysis was performed until the patients died or end of the research. Patients were divided into two groups depending on NACK expression level (low and high). We observed that the cumulative 4-year overall survival rate (OS) and decreased survival rate (DFS) of patients with high level of NACK detection were 30.7 and $34.6 \%$, respectively, when compared to low NACK expression patients (77.8 and 77.8\%, respectively) (Fig. 2). Hence, the level of NACK expression was an unfavorable predictive factor for prognosis of NSCLC patients.

Knockdown of NACK inhibits the expression of NACK in NSCLC cells. The efficient inhibition of NACK expression was examined by qRT-PCR and western blotting, the result showed that after transfected with NACK-siRNA, the expression of NACK was significantly decreased at mRNA level (Fig. 3A) and hardly observed at protein level (Fig. 3B) either in A549 or H1299 cells. No significant difference was observed between negative-siRNA and control groups.

NACK is required for cell proliferation in NSCLC. Cell proliferation assay and cell invasion assay were performed in NSCLC cell lines A549 and H1299 with NACK knockdown by siRNA. CCK-8 assay verified that the cells transfected with NACK-siRNA showed a significant decrease in cell proliferation when compared with control group both in A549 

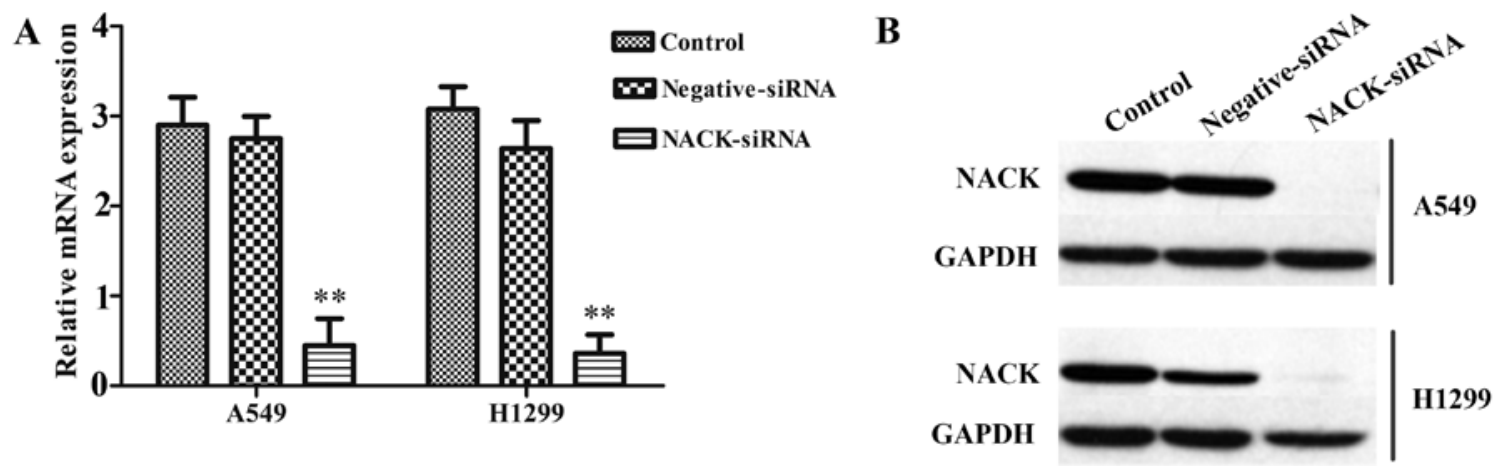

Figure 3. Silencing of NACK suppresses the expression level of NACK in NSCLC cells. (A) mRNA levels of NACK in NACK knockdown cells detected by qRT-PCR. (B) Protein levels of NACK in NACK knockdown cells detected by western blot analysis. GAPDH was used as internal control. Data shown as mean \pm SD of triplicate independent experiments. ${ }^{* *} \mathrm{p}<0.01$ when compared to negative-siRNA group.

A
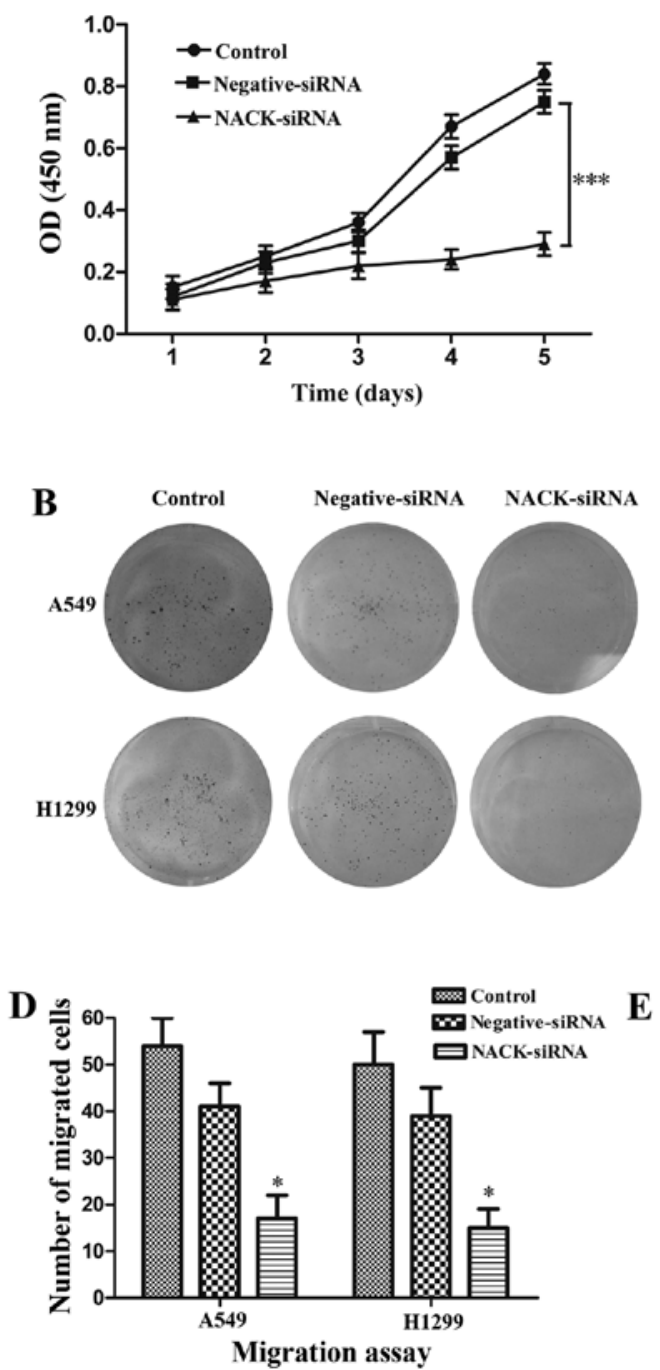

H1299 cells
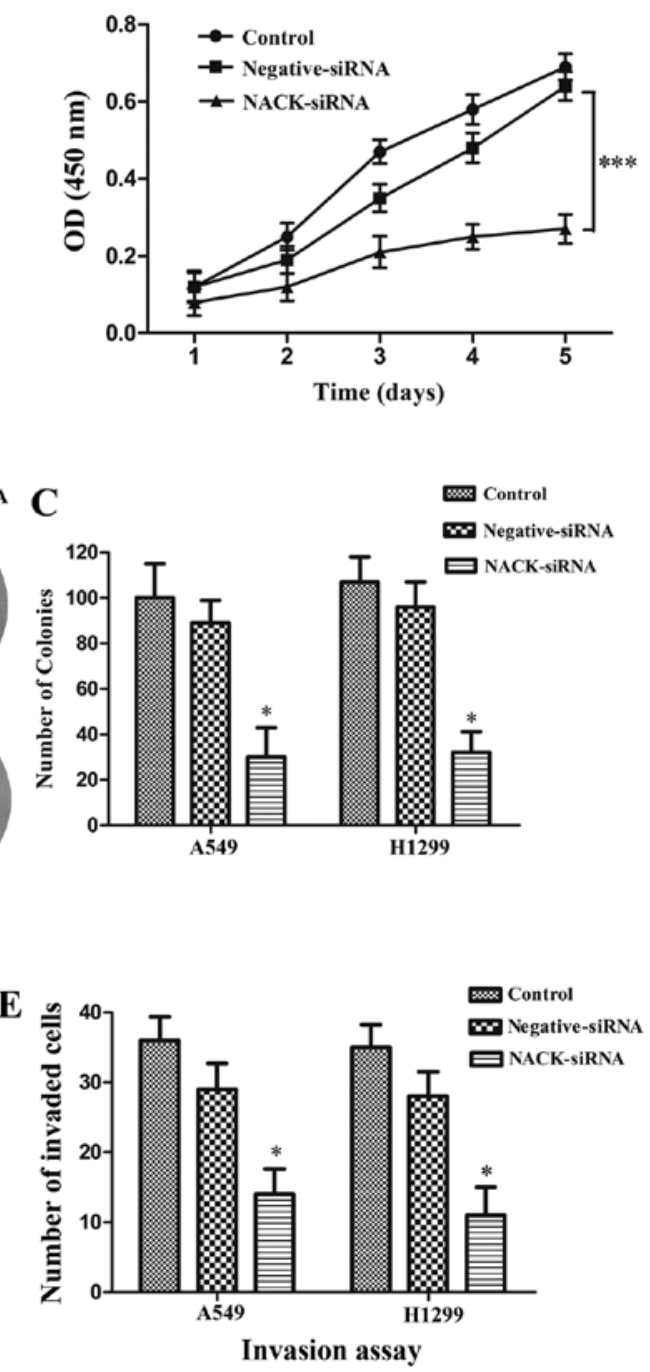

Figure 4. Knockdown of NACK inhibited NSCLC cell proliferation and motility in vitro. (A) NACK-siRNA inhibited the proliferation of A549 and H1299 cells detected by CCK-8. (B) Photographs of colonies with Giemsa staining from A549 and H1299 cells treated with NACK silencing. (C) Statistical analysis of the number of colonies in A549 and H1299 cells with silenced NACK. (D) Effect of NACK silencing on the migration of A549 and H1299 cells. (E) Effect of NACK silencing on the invasion of A549 and H1299 cells. Data shown are the mean \pm SD of triplicate independent experiments. ${ }^{*} \mathrm{p}<0.05$ and ${ }^{* * * *} \mathrm{p}<0.001$ when compared to negative-siRNA group.

$(\mathrm{p}<0.001)$ and $\mathrm{H} 1299(\mathrm{p}<0.001)$ cells at the 5th day $($ Fig. 4A). The colony formation assay was used to evaluate the tumor growth of a transfected cells. Our data indicated that when A549 and H1299 cells were treated with NACK-siRNA, the 

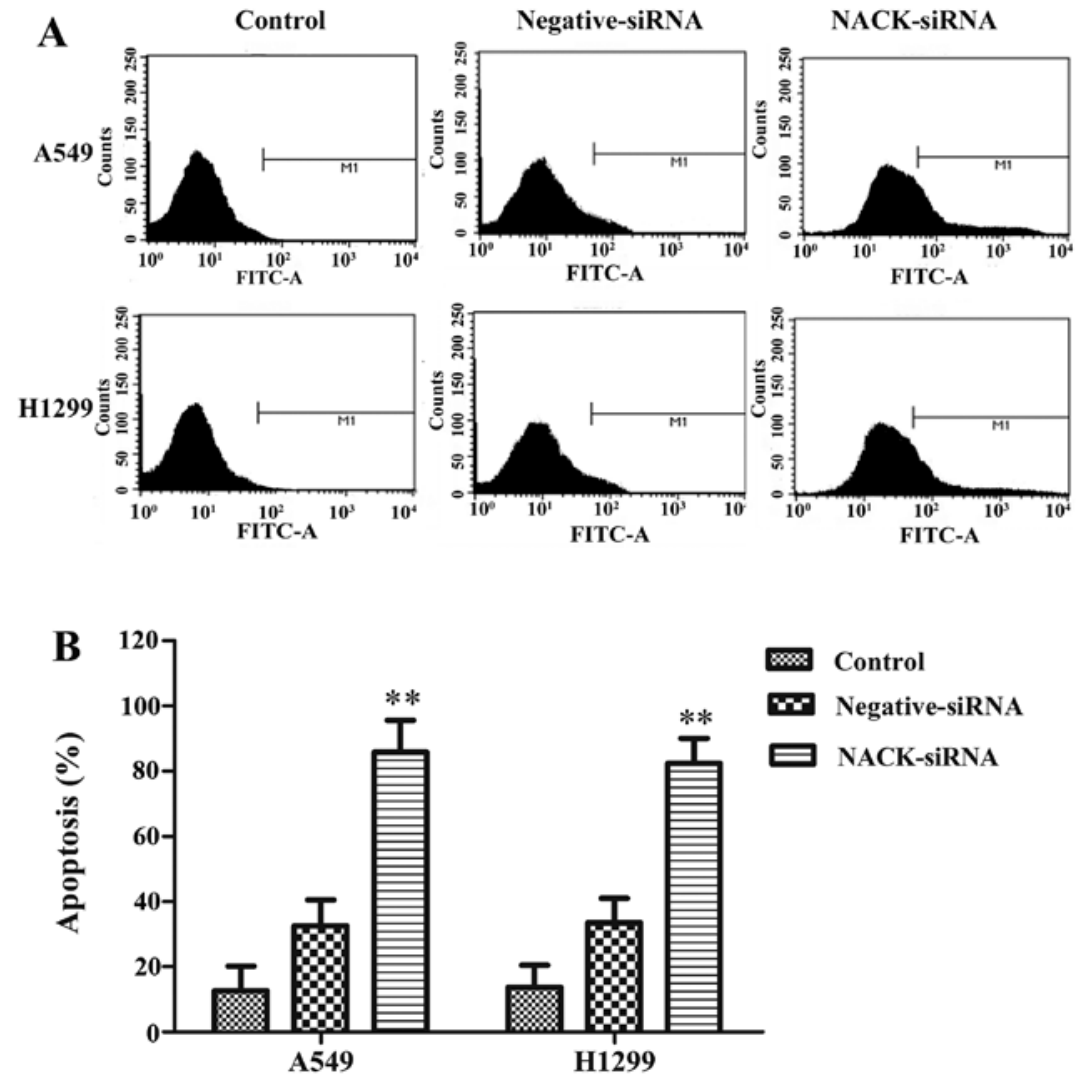

Figure 5. The effect of NACK silencing on cell apoptosis of A549 and H1299 cells. (A) Flow cytometric analysis of A549 and H1299 cells transfected with NACK-siRNA. (B) Percentage of apoptotic cells. Data are shown as the mean \pm SD of triplicate independent experiments. ${ }^{* *} \mathrm{p}<0.01$ compared to the negative-siRNA group.

ability of colony formation was remarkably decreased in A549 $(\mathrm{p}<0.05)$ and H1299 (p<0.05) cells (Fig. 4B and C).

NACK knockdown inhibits the migration and invasion of NSCLC cells. To investigate whether NACK regulates the migration and invasion of NSCLC cell, NSCLC cells were transfected with either NACK-siRNA or negative-siRNA. Cell migration and invasion were evaluated by using a Transwell insert. The results demonstrated that knockdown of NACK significantly reduced the number of migration at $24 \mathrm{~h}(\mathrm{p}<0.01)$ and invasion at $48 \mathrm{~h}(\mathrm{p}<0.01)$ (Fig. $4 \mathrm{D}$ and $\mathrm{E})$, suggesting that NACK is necessary for NSCLC cell metastasis during the tumor progress.

Induction of apoptosis by interference of NACK. We further investigated whether interference NACK could induce apoptosis in A549 and H1299 cells. FITC assay results indicated that the apoptosis rates were significantly increased both in A549 and H1299 cells after knocking down NACK (Fig. 5). These results suggest the interference of NACK inhibits NSCLC progression through apoptosis induction in vitro.

Knocking down NACK inhibits the transcriptional activity of Notch signaling pathway in NSCLC. NACK was identified as a direct target gene of Notch pathway in human esophageal adenocarcinoma cells (15). Considering the specific action of the Notch pathway in different types of tumors, we investigated the role of NACK on the regulation of Notch pathway in NSCLC by transfecting NACK-siRNA into A549 and H1299 cells to knock down NACK expression. The results showed that the expression of the Notch target genes Hes1 and HeyL were reduced significantly at mRNA level (Fig. 6A). Western blot results also showed that transfection with NACK-siRNA reduced expression of Notch1, Hes1 and HeyL protein both in A549 and H1299 cells (Fig. 6B). Furthermore, as luciferase reporter assay result showed, co-transfection of NACK-siRNA with Hes1 reporter construct strongly reduced luciferase activity in both A549 and H1299 cells (Fig. 6C) when compared to the cells co-transfected with negative-siRNA. The data demonstrated that the transcriptional activity of Notch1 signaling pathway could be inhibited by knocking down the expression of NACK in NSCLC.

Interference of NACK inhibits NSCLC progression in vivo. To address whether changes of NACK expression could influence the growth of tumor in vivo, A549 and H1299 cells infected with negative-siRNA or NACK-siRNA were injected subcutaneously into the nude mice, and tumor size was measured every 4 days for 28 days. The results revealed that the knockdown of NACK inhibited tumorigenesis in vivo. The average tumor volume (Fig. 7A) and weight of mice (Fig. 7B and C) in NCAK-siRNA group at day 28 was significantly decreased $(p<0.01)$ compared to that of mice in control group. These results demonstrated that the interference of NACK inhibits NSCLC progression in vivo. 

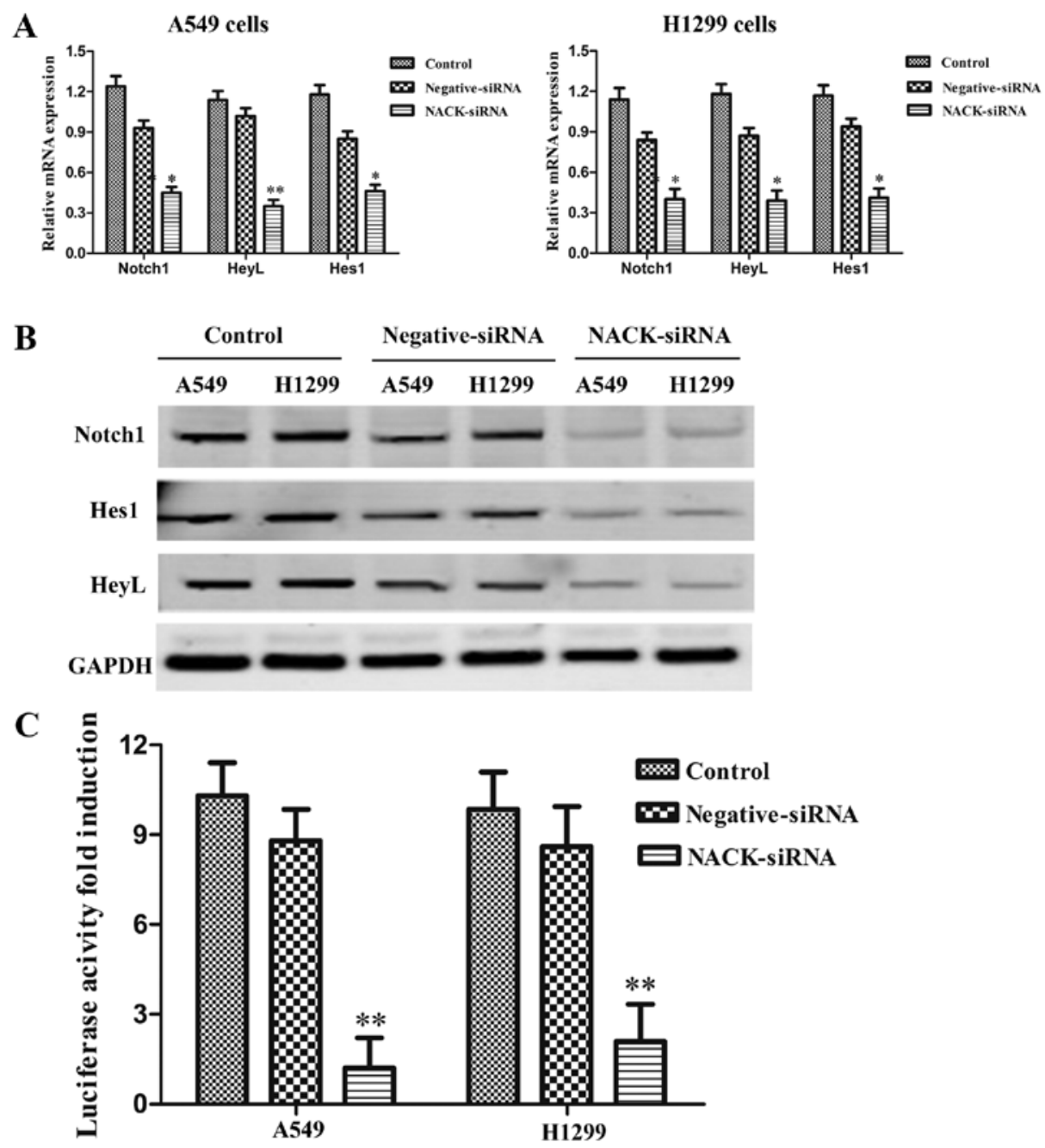

Figure 6. NACK is a direct target of Notch1 signaling pathway. (A) The relative mRNA levels of Notch1 signaling pathway-related genes detected by qRT-PCR (B) The expressions of Notch1 signaling pathway-related proteins detected by western blot analysis. (C) Luciferase activity analysis of Hes1 reporter after NACK silencing. GAPDH was used as internal control. ${ }^{*} p<0.05$ and ${ }^{* *} \mathrm{p}<0.01$ when compared to negative-siRNA group.

\section{Discussion}

Notch signaling is an evolutionarily conserved intercellular communication mechanism critical for cell survival, proliferation, differentiation, as well as maintaining stem cell quiescence and identity (16-18). Moreover, it has great relevance to multiple aspects of cancer biology, from cancer stem cells, angiogenesis to tumor immunity (19-23). Jiang et al, observed strong Notch-1 immunoreactivity in NSCLC, which correlated with Jagged-1 and VEGF expression (24). Under hypoxia, Notch-1 provides important survival signals to NSCLC cells (25). Baumgart et al, reported that Notch-1 also acts as a regulator of EGFR expression through incorporating with ADAM17 in NSCLC (26). Ji et al, discovered that Notch-1 downregulation inhibited cell growth and induced apoptosis in NSCLC by $\delta$-tocotrienol (27). Furthermore, Notch-1 promoted NSCLC tumor progression through direct up-regulation of insulin-like growth factor 1 receptor (IGF1-R) (28) facilitating expression of the survivin (29).

NACK, a kind of atypical kinase (30), was first named Pragmin due to its ability to stimulate the activity of RhoA (31). Recently, Weaver et al, reported the crucial role of
NACK as a novel regulator of Notch transcription and as the Notch-mediated tumor proliferation of mammary epithelial cells and esophageal adenocarcinoma cells (15). Therefore, we hypothesized that interference of NACK may inhibit the progression of NSCLC. Thus, the relationship between NACK expression and tumorigenesis of NSCLC is first demonstrated in this study.

Through detecting the expression of NACK in 35 tumor samples from NSCLC patients and analyzing their clinicopathological parameters, we found that NACK was remarkably over-expressed in NSCLC tumor tissues both at transcriptional and translational levels. Furthermore, high NACK expression was associated with tumor differentiation, lymphatic metastasis, clinical stage and poor survival prognosis in NSCLC patients, which indicated that NACK may be an independent prognostic factor for NSCLC.

RNA interference method as a powerful technology (32) was used in this study to knock down NACK expression. Cell indefinite proliferation, invasion and metastasis are the major causes of NSCLC occurrence (33). From in vivo and in vitro studies, we found that the interference of NACK markedly inhibited the proliferation, invasion and metastasis of NSCLC cells, indicating that NACK is necessary for the prolif- 
A
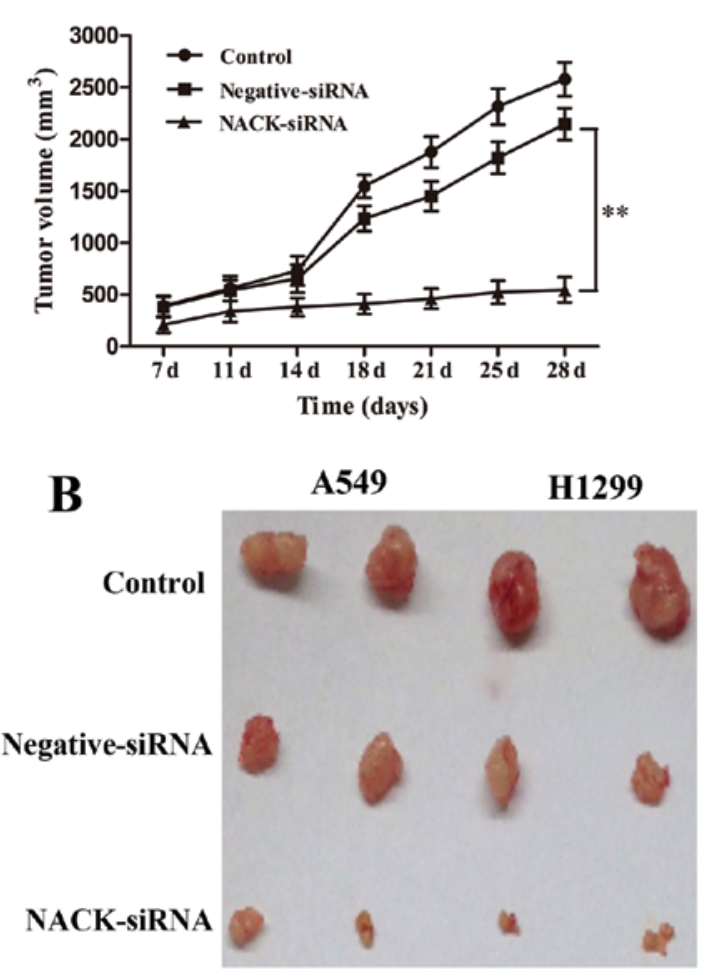

H1299 cells

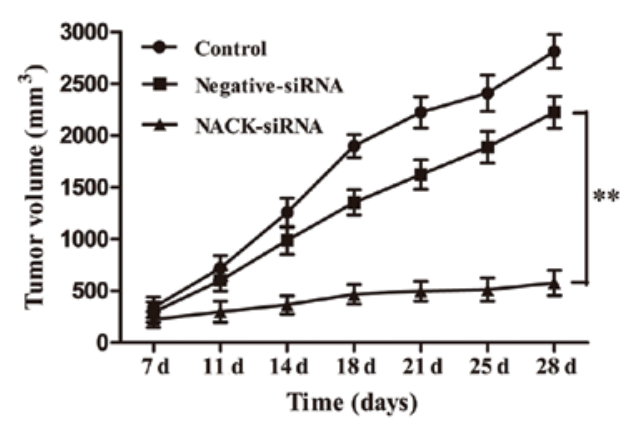

C

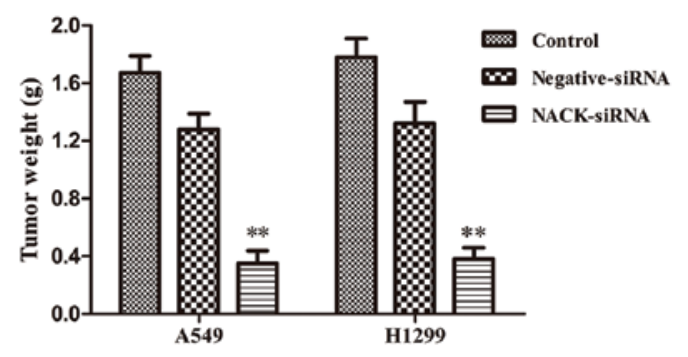

Figure 7. NACK silencing inhibited tumorigenesis in vivo. (A) The curve of average tumor volume in nude mouse inoculation with A549 and H1299 cells treated with NACK-siRNA during the 28-day observation. (B) Representative images of tumors separated from each mouse group on day 28. (C) Average tumor weight in each group of nude mice on day $28 .{ }^{* *} \mathrm{p}<0.01$ when compared to negative-siRNA group.

eration of tumor cells, which was consistent with a previous study (15). Apoptosis plays a vital role in cancer development by the dysregulation of cell death and cell growth (34). Based on the flow cytometry assay, the interference of NACK significantly induces apoptosis rate in NSCLC cells, demonstrating that NACK may be a potential agent for the treatment of NSCLC $(35,36)$.

Hes1 and HeyL, members of Hes and Hey families, respectively, have been reported as the direct downstream targets of the Notch1 signaling (37-39). In the present study, we found that their expression was markedly reduced when NACK was knocked down. Moreover, luciferase reporter assay also showed down-regulation of Notch1 signaling with NACK silencing in NSCLS cells. The Notch1 target genes Hes1 and HeyL are involved in regulating cell proliferation, apoptosis, differentiation and metabolism $(40,41)$. Taken together, we hypothesized that the interference of NACK by siRNA inhibits tumorigenesis of NSCLC directly via targeting the Notch1 signaling pathway. However, the mechanisms of interference of NACK inhibiting tumorigenesis is not clear, and further research is necessary.

In conclusion, we provide the first evidence to demonstrate that NACK was robustly expressed in a subset of NSCLC samples and interference of NACK inhibits NSCLC progression through failing to activate Notch1 signaling complexes. Thus, NACK may be an attractive target to help develop novel therapeutic methods against NSCLC. Insightful studies are still needed to disclose other signaling pathways involved in regulating NSCLC.

\section{Acknowledgements}

We would like to thank Dr Xijing He and Dr Bin Zhou for their insightful comments and suggestions.

\section{References}

1. Siegel R, Ma J, Zou Z and Jemal A: Cancer statistics, 2014. CA Cancer J Clin 64: 9-29, 2014.

2. Nguyen KS, Neal JW and Wakelee H: Review of the current targeted therapies for non-small-cell lung cancer. World J Clin Oncol 5: 576-587, 2014.

3. Boolell V, Alamgeer M, Watkins DN and Ganju V: The evolution of therapies in non-small cell lung cancer. Cancers (Basel) 7: 1815-1846, 2015.

4. Rovigatti U: Cancer modelling in the NGS era - Part I: Emerging technology and initial modelling. Crit Rev Oncol Hematol 96: 274-307, 2015.

5. Gottfried M, Keizman D, Mishaeli M and Rabinovich NM: The current approach to advanced lung cancer. Harefuah 154: 521-540, 2015 (In Hebrew).

6. Wang Z, Li Y, Banerjee S and Sarkar FH: Emerging role of Notch in stem cells and cancer. Cancer Lett 279: 8-12, 2009.

7. Kristoffersen K, Villingshøj M, Poulsen HS and Stockhausen MT: Level of Notch activation determines the effect on growth and stem cell-like features in glioblastoma multiforme neurosphere cultures. Cancer Biol Ther 14: 625-637, 2013.

8. Jang MS, Miao H, Carlesso N, Shelly L, Zlobin A, Darack N, Qin JZ, Nickoloff BJ and Miele L: Notch-1 regulates cell death independently of differentiation in murine erythroleukemia cells through multiple apoptosis and cell cycle pathways. J Cell Physiol 199: 418-433, 2004

9. Li L, Zhao F, Lu J, Li T, Yang H, Wu C and Liu Y: Notch-1 signaling promotes the malignant features of human breast cancer through NF- $\kappa$ B activation. PLoS One 9: e95912, 2014.

10. Leong KG and Karsan A: Recent insights into the role of Notch signaling in tumorigenesis. Blood 107: 2223-2233, 2006. 
11. Leong KG and Gao WQ: The Notch pathway in prostate development and cancer. Differentiation 76: 699-716, 2008.

12. Miele L and Osborne B: Arbiter of differentiation and death: Notch signaling meets apoptosis. J Cell Physiol 181: 393-409, 1999.

13. Wang Z, Li Y, Banerjee S and Sarkar FH: Exploitation of the Notch signaling pathway as a novel target for cancer therapy. Anticancer Res 28: 3621-3630, 2008.

14. Yin L, Velazquez OC and Liu ZJ: Notch signaling: Emerging molecular targets for cancer therapy. Biochem Pharmacol 80 690-701, 2010

15. Weaver KL, Alves-Guerra MC, Jin K, Wang Z, Han X, Ranganathan P, Zhu X, DaSilva T, Liu W, Ratti F, et al: NACK is an integral component of the Notch transcriptional activation complex and is critical for development and tumorigenesis Cancer Res 74: 4741-4751, 2014.

16. Androutsellis-Theotokis A, Leker RR, Soldner F, Hoeppner DJ, Ravin R, Poser SW, Rueger MA, Bae SK, Kittappa R and McKay RD: Notch signalling regulates stem cell numbers in vitro and in vivo. Nature 442: 823-826, 2006.

17. Bi P and Kuang S: Notch signaling as a novel regulator of metabolism. Trends Endocrinol Metab 26: 248-255, 2015.

18. Guruharsha KG, Kankel MW and Artavanis-Tsakonas S: The Notch signalling system: Recent insights into the complexity of a conserved pathway. Nat Rev Genet 13: 654-666, 2012.

19. Galluzzo P and Bocchetta M: Notch signaling in lung cancer. Expert Rev Anticancer Ther 11: 533-540, 2011.

20. Nickoloff BJ, Osborne BA and Miele L: Notch signaling as a therapeutic target in cancer: A new approach to the development of cell fate modifying agents. Oncogene 22: 6598-6608, 2003.

21. Ranganathan P, Weaver KL and Capobianco AJ: Notch signalling in solid tumours: A little bit of everything but not all the time. Nat Rev Cancer 11: 338-351, 2011.

22. Takebe N, Harris PJ, Warren RQ and Ivy SP: Targeting cancer stem cells by inhibiting Wnt, Notch, and Hedgehog pathways. Nat Rev Clin Oncol 8: 97-106, 2011.

23. Wael H, Yoshida R, Kudoh S, Hasegawa K, Niimori-Kita K and Ito T: Notch1 signaling controls cell proliferation, apoptosis and differentiation in lung carcinoma. Lung Cancer 85: 131-140, 2014.

24. Jiang X, Zhou JH, Deng ZH, Qu XH, Jiang HY and Liu Y: Expression and significance of Notch1, Jagged1 and VEGF in human non-small cell lung cancer. Zhong Nan Da Xue Xue Bao Yi Xue Ban 32: 1031-1036, 2007 (In Chinese).

25. Chen Y, De Marco MA, Graziani I, Gazdar AF, Strack PR, Miele L and Bocchetta M: Oxygen concentration determines the biological effects of NOTCH-1 signaling in adenocarcinoma of the lung. Cancer Res 67: 7954-7959, 2007.

26. Baumgart A, Seidl S, Vlachou P, Michel L, Mitova N, Schatz N, Specht K, Koch I, Schuster T, Grundler R, et al: ADAM17 regulates epidermal growth factor receptor expression through the activation of Notch 1 in non-small cell lung cancer. Cancer Res 70: 5368-5378, 2010.
27. Ji X, Wang Z, Geamanu A, Sarkar FH and Gupta SV: Inhibition of cell growth and induction of apoptosis in non-small cell lung cancer cells by delta-tocotrienol is associated with notch-1 down-regulation. J Cell Biochem 112: 2773-2783, 2011.

28. Eliasz S, Liang S, Chen Y, De Marco MA, Machek O, Skucha S, Miele L and Bocchetta M: Notch-1 stimulates survival of lung adenocarcinoma cells during hypoxia by activating the IGF-1R pathway. Oncogene 29: 2488-2498, 2010.

29. Chen Y, Li D, Liu H, Xu H, Zheng H, Qian F, Li W, Zhao C, Wang $Z$ and Wang $X$ : Notch-1 signaling facilitates survivin expression in human non-small cell lung cancer cells. Cancer Biol Ther 11: 14-21, 2011.

30. Manning G, Whyte DB, Martinez R, Hunter T and Sudarsanam S: The protein kinase complement of the human genome. Science 298: 1912-1934, 2002.

31. Tanaka H, Katoh $\mathrm{H}$ and Negishi M: Pragmin, a novel effector of Rnd2 GTPase, stimulates RhoA activity. J Biol Chem 281: 10355-10364, 2006.

32. Nguyen TA and Fruehauf JH: Transkingdom RNA interference (tkRNAi): A novel method to induce therapeutic gene silencing. Methods Mol Biol 514: 27-34, 2009.

33. Geng J, Li X, Zhou Z, Wu CL, Dai M and Bai X: EZH2 promotes tumor progression via regulating VEGF-A/AKT signaling in non-small cell lung cancer. Cancer Lett 359: 275-287, 2015.

34. Koff JL, Ramachandiran S and Bernal-Mizrachi L: A time to kill: Targeting apoptosis in cancer. Int J Mol Sci 16: 2942-2955, 2015.

35. Drosopoulos K and Pintzas A: Multifaceted targeting in cancer: The recent cell death players meet the usual oncogene suspects. Expert Opin Ther Targets 11: 641-659, 2007.

36. Taylor K, Micha D, Ranson M and Dive C: Recent advances in targeting regulators of apoptosis in cancer cells for therapeutic gain. Expert Opin Investig Drugs 15: 669-690, 2006.

37. Fischer A and Gessler M: Delta-Notch - and then? Protein interactions and proposed modes of repression by Hes and Hey bHLH factors. Nucleic Acids Res 35: 4583-4596, 2007.

38. Nakagawa O, McFadden DG, Nakagawa M, Yanagisawa H, Hu T, Srivastava D and Olson EN: Members of the HRT family of basic helix-loop-helix proteins act as transcriptional repressors downstream of Notch signaling. Proc Natl Acad Sci USA 97: 13655-13660, 2000.

39. Iso T, Kedes L and Hamamori Y: HES and HERP families: Multiple effectors of the Notch signaling pathway. J Cell Physiol 194: 237-255, 2003.

40. Al-Hussaini H, Subramanyam D, Reedijk M and Sridhar SS: Notch signaling pathway as a therapeutic target in breast cancer. Mol Cancer Ther 10: 9-15, 2011.

41. Candy PA, Phillips MR, Redfern AD, Colley SM, Davidson JA, Stuart LM, Wood BA, Zeps N and Leedman PJ: Notch-induced transcription factors are predictive of survival and 5-fluorouracil response in colorectal cancer patients. Br J Cancer 109: 10231030, 2013. 